\title{
Search for encapsulation of platinum, silver, and gold at the surface of graphite
}

\author{
Ann Lii-Rosales, ${ }^{1,2, *}$ Yong Han,,${ }^{1,3}$ Dapeng Jing $\odot,{ }^{1}$ Michael C. Tringides,,${ }^{1,3}$ and Patricia A. Thiel $\circledast^{1,2,4, \dagger}$ \\ ${ }^{1}$ Ames Laboratory of the USDOE, Ames, Iowa 50011, USA \\ ${ }^{2}$ Department of Chemistry, Iowa State University, Ames, Iowa 50011, USA \\ ${ }^{3}$ Department of Physics and Astronomy, Iowa State University, Ames, Iowa 50011, USA \\ ${ }^{4}$ Department of Materials Science and Engineering, Iowa State University, Ames, Iowa 50011, USA
}

(Received 20 March 2020; revised 19 June 2020; accepted 30 June 2020; published 31 July 2020)

\begin{abstract}
Using scanning tunneling microscopy, we show that Pt clusters can be encapsulated beneath the surface of graphite, whereas $\mathrm{Ag}$ and $\mathrm{Au}$ cannot. This is in complete agreement with independent predictions from density functional theory, which show that surface intercalation of single metal atoms is favorable for Pt, but unfavorable for $\mathrm{Ag}$ and $\mathrm{Au}$. This supports the validity of using single-metal-atom energetics for predicting encapsulation of metal nanoparticles at the graphite surface. We also demonstrate that the optimal temperature for encapsulation scales with the cohesive energy of the metal.
\end{abstract}

DOI: 10.1103/PhysRevResearch.2.033175

\section{INTRODUCTION}

Many applications of solid metals - as catalysts, magnets, sensors, heat sinks, or electrodes, to name a few-are most efficient and cost effective when the surface-to-volume ratio of the metal is high. Recently, we have reported that high surface-to-volume ratios in metals can be achieved by encapsulating metal nanoclusters at the surface of a layered material, graphite. Effectively, the layered material resists deformation, thereby forcing the metal cluster between the layers to adopt a much flatter profile (higher aspect ratio) than it would otherwise [1,2]. Meanwhile the valuable properties of the metal may remain accessible or even be enhanced, e.g., in catalysis [3,4], magnetism [5], photonics [6], or other applications, despite being covered by a graphene layer(s).

The question then naturally emerges, which metals can be encapsulated at the surface of graphite? We have found that transition metals $\mathrm{Cu}, \mathrm{Ru}$, and $\mathrm{Fe}$, as well as the rare earth Dy, can be embedded, provided two conditions are met in synthesizing the metal-plus-graphite surface system [7-10]. First, defects must be created on the clean graphite surface, to provide entry portals for the metal [11]. We achieve this by ion sputtering with argon. Second, metal must be deposited on the defect-rich graphite surface at elevated temperature, rather than being deposited at low temperature and heated. We believe the elevated temperature is necessary to prevent

\footnotetext{
* Present address: Department of Chemistry, University of Colorado Boulder, Boulder, CO 80309, USA.

†pthiel@ameslab.gov
}

Published by the American Physical Society under the terms of the Creative Commons Attribution 4.0 International license. Further distribution of this work must maintain attribution to the author(s) and the published article's title, journal citation, and DOI. the portals from becoming blocked by metal atoms [11], which have a high affinity for undercoordinated carbon atoms such as those at steps and defects [12,13]. Dissolution of metal clusters at portals engenders an expectation that the temperature required for encapsulation should scale, at least roughly, with the cohesive energy of the metal [9].

The observation of surface encapsulation of these metals is somewhat surprising, given that there is no precedent for the transition metals $(\mathrm{Cu}, \mathrm{Ru}, \mathrm{Fe})$ to form bulk graphite intercalation compounds [14]. In the one case we have studied where precedent does exist - for the rare earth Dy [14]—features of Dy clusters enclosed at the graphite surface depart significantly from those known for Dy enclosed in the graphite bulk. One main difference is that Dy clusters at the surface consist of three metal layers, whereas a Dy layer in the intercalation compound does not exceed a single layer in height [7]. The transition metals can form even taller surface-encapsulated clusters, up to 200 metal layers for $\mathrm{Cu}$ [8]. Clearly, the forces and processes that lead to surface encapsulation are different than those that would lead to bulk intercalation.

We have, in fact, conjectured that the formation of encapsulated clusters may be driven, in part or in whole, by surface kinetics rather than thermodynamics (depending on the metal). This point is made most clearly in the $\mathrm{Cu}$ /graphite system, where density functional theory (DFT) showed that there is no driving force for encapsulation of $\mathrm{Cu}$ multilayers, despite the experimental observation of very large embedded $\mathrm{Cu}$ nanoclusters. However, DFT showed that there is a large driving force for encapsulation of single $\mathrm{Cu}$ atoms [8]. This would lead to a high population of $\mathrm{Cu}$ atoms in the gallery beneath the topmost graphene monolayer (GML). These $\mathrm{Cu}$ atoms could then nucleate and become trapped in the gallery.

In this paper we extend the investigations to three more transition metals: $\mathrm{Pt}, \mathrm{Ag}$, and $\mathrm{Au}$. Pt nanoclusters are, of course, of general interest due to the broad catalytic properties of Pt [15]. Ag and Au nanoclusters generate interest, not only 
because of their catalytic properties [16,17], but also because of their useful photonic properties [18,19]. We show that $\mathrm{Pt}$ nanoclusters can be embedded in the graphite surface, but $\mathrm{Au}$ and Ag cannot. This is completely supported by our DFT calculations, which indicate that single atoms of $\mathrm{Pt}$ are stable beneath the topmost GML, whereas Au and Ag atoms are not.

This paper is organized as follows. Section II provides a summary of experimental methods and computational methodology, benchmarking, and energy definitions. Sections III and IV present experimental data and DFT results, respectively, for all three metals on/in graphite. Section $\mathrm{V}$ is a discussion, and Sec. VI concludes the paper.

\section{EXPERIMENTAL AND COMPUTATIONAL METHODS}

\section{A. Experimental methods}

Experiments were performed in an Omicron ultrahighvacuum (UHV) chamber with base pressure of $2 \times 10^{-11}$ mbar. In short, the metals were deposited via physical vapor deposition from an $e$-beam evaporator onto commercially available highly oriented pyrolytic graphite (HOPG, ZYB grade). The graphite surface was either pristine (cleaved using Scotch tape; referred to as $p$-graphite hereafter) or defect rich (bombarded with argon ions; referred to as $i$-graphite hereafter). Metals were deposited onto either type of surface while the graphite sample was held at different temperatures, $T_{\text {dep }}$. Major characterization techniques included scanning tunneling microscopy (STM; images were acquired in constant-current mode) and X-ray photoelectron spectroscopy (XPS), both performed with the sample at room temperature. More experimental details are available in the Supplemental Material [20].

\section{B. Computational methods}

DFT methodology. We perform first-principles DFT total energy calculations for the Ag-, Au-, and Pt-graphite systems using the plane-wave VASP code [21]. The projectoraugmented-wave (PAW) method [22] is used for the electroncore interactions, and the optB88-vdW functional, where the exchange functional is optimized for the correlation part [23], is used to approximately account for dispersion interactions. The pseudopotentials were generated and released in 2013 by the VASP group. Spin-polarization effects and dipole corrections have been taken into account in all DFT calculations. The $\Gamma$-centered $k$ mesh will be specified for each type of system to be calculated.

In calculations for various metal-plus-graphite systems, we always use a graphite slab consisting of 4 GMLs as the substrate with a $2-\mathrm{nm}$ vacuum thickness along the direction perpendicular to the slab surface, and the lateral size of the supercell is taken to be $6 \times 6$ in units of the lateral lattice constant of graphite, $a_{\mathrm{C}}$. During relaxation for energy minimization, the bottom-most GML of the substrate is fixed. The $k$ mesh is taken to be $7 \times 7 \times 1$, and the energy cutoff is $600 \mathrm{eV}$. The force-convergence criterion is $0.1 \mathrm{eV} / \mathrm{nm}$. All these parameters have been carefully tested for energy convergence.

Benchmarking. We have carried out benchmark calculations for the single-phase systems graphite, $\mathrm{Pt}, \mathrm{Ag}$, and $\mathrm{Au}$.
For graphite, from our optB88-vdW calculation, we obtain a lattice constant of $a_{\mathrm{C}}=0.2465 \mathrm{~nm}$ (cf. experimental value $a_{\mathrm{C}}=0.246 \mathrm{~nm}$ [24]). For more information, see Ref. [25]. For the three metals, in these benchmark calculations, we consistently use a $k$ mesh of $61 \times 61 \times 61$ for the primitive unit cell and an energy cutoff of $600 \mathrm{eV}$. For fcc Ag, we calculate $a_{\mathrm{Ag}}=0.4132 \mathrm{~nm}$ (cf. experimental value $a_{\mathrm{Ag}}=0.4069 \mathrm{~nm}$ extrapolated to $0 \mathrm{~K}$ [26]), and cohesive energy of $2.821 \mathrm{eV}$ (cf. experimental value $2.95 \mathrm{eV}$ [27]). For fcc Au, we obtain a lattice constant of $a_{\mathrm{Au}}=0.4161 \mathrm{~nm}$ (cf. experimental value $a_{\mathrm{Au}}=0.4065 \mathrm{~nm}$ extrapolated to $0 \mathrm{~K}$ [26]), and cohesive energy of $3.404 \mathrm{eV}$ (cf. experimental value $3.81 \mathrm{eV}$ [27]). For fcc Pt, we obtain $a_{\mathrm{Pt}}=0.3980 \mathrm{~nm}$ (cf. experimental value $a_{\mathrm{Pt}}=0.39160 \mathrm{~nm}$ extrapolated to $0 \mathrm{~K}$ [28]), and cohesive energy of $5.817 \mathrm{eV}$ (cf. experimental value $5.84 \mathrm{eV}$ [27]). In short, the agreement between theory and experiment is very good.

Energy definitions. To determine whether it is favorable for a metal cluster with $n$ atoms to be adsorbed on (top of) a graphite surface or intercalated underneath the top GML, we define the chemical potential $\mu_{M}(M=\mathrm{Pt}, \mathrm{Ag}$, or $\mathrm{Au})$ of the metal cluster as

$$
\mu_{M}=\frac{E_{\text {tot }}-E_{\text {graphite }}}{n}-E_{M},
$$

where $E_{\mathrm{tot}}$ is the total energy of the metal-plus-graphite system, $E_{\text {graphite }}$ is the energy of the graphite substrate, and $E_{M}$ is the energy of one metal atom in the gas phase. For one adatom $(n=1)$ adsorbed on the substrate, Eq. (1) is reduced to the conventional expression for the adsorption energy $E_{\text {ads }}=E_{\text {tot }}-E_{\text {graphite }}-E_{M}$.

\section{EXPERIMENTAL RESULTS}

\section{A. Characteristics of graphite surfaces: General comments}

Detailed characterization and descriptions of $p$ - and $i$ graphite surfaces are available elsewhere [7,8]. In short, $p$-graphite has flat and smooth terraces. Triangular arrays of $\mathrm{C}$ atoms (three out of six atoms in each hexagon) can be resolved, with lattice spacing of $0.247 \pm 0.003 \mathrm{~nm}$. This agrees well with the literature value of graphite lattice spacing of $0.246 \mathrm{~nm}$ [24]. After subjecting $p$-graphite to ion bombardment, surface defects appear as bright but rather diffuse protrusions with height $\leqslant 0.35 \mathrm{~nm}$. STM reveals that defects come in a variety of shapes and sizes. Some are similar to single-atom vacancies with threefold symmetry [29-31] [Figs. 1(a) and 1(b)]. Other types presumably include multiatom vacancies and interstitial $\mathrm{C}$ atoms that are displaced within the graphite gallery (the space between graphene layers) as a result of ion bombardment [31-33] [Figs. 1(c) and $1(\mathrm{~g})]$. Evidence of electronic perturbation is often present around defects, manifest as a well-known $(\sqrt{3} \times \sqrt{3}) R 30^{\circ}$ superlattice [34,35] [Figs. 1(e) and 1(f)]. Examples of these features are also shown in Refs. $[8,10]$.

\section{B. Temperature dependence: General comments}

We have demonstrated that bare (unencapsulated) metal clusters predominate at low $T_{\mathrm{dep}}$, then diminish in population 

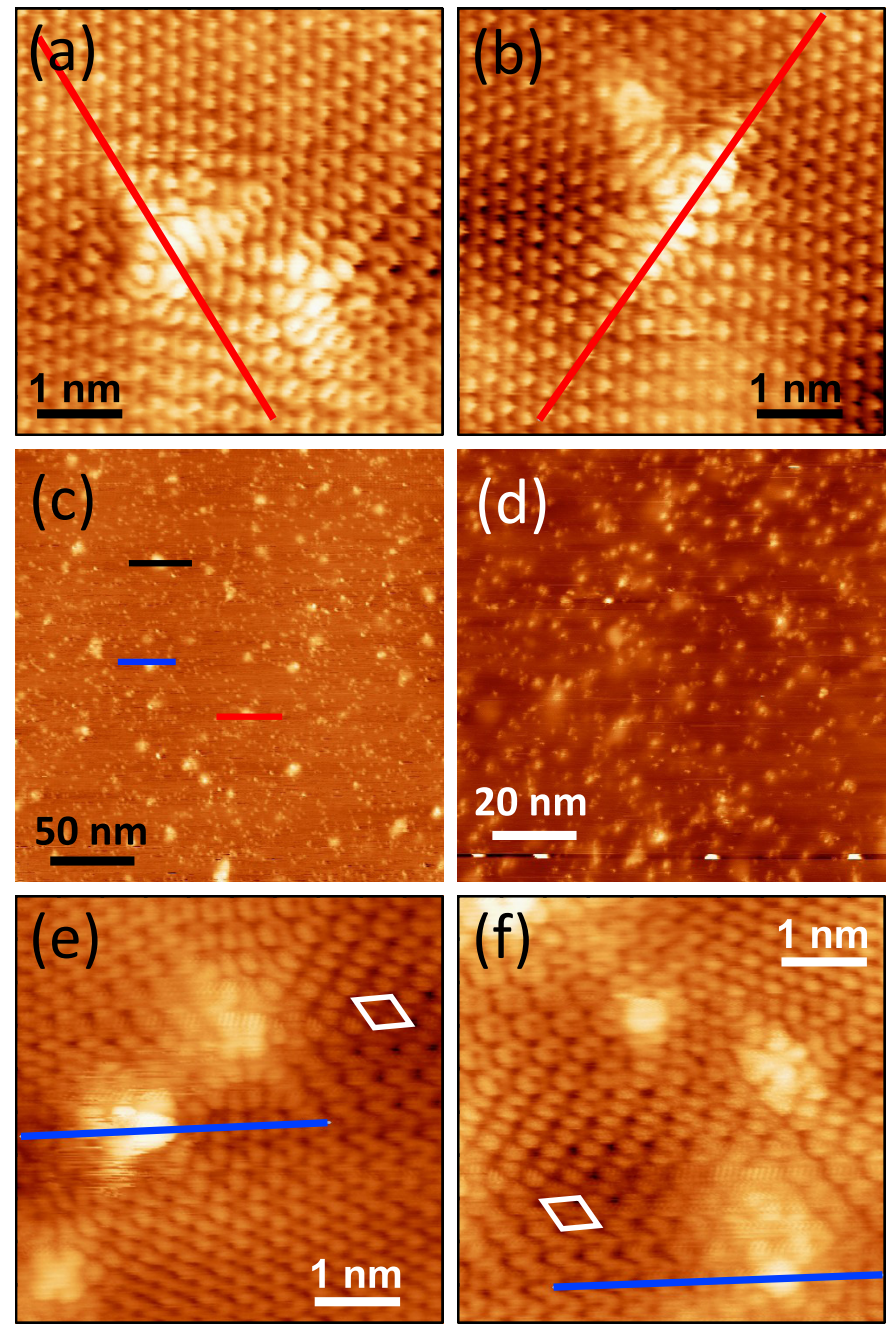
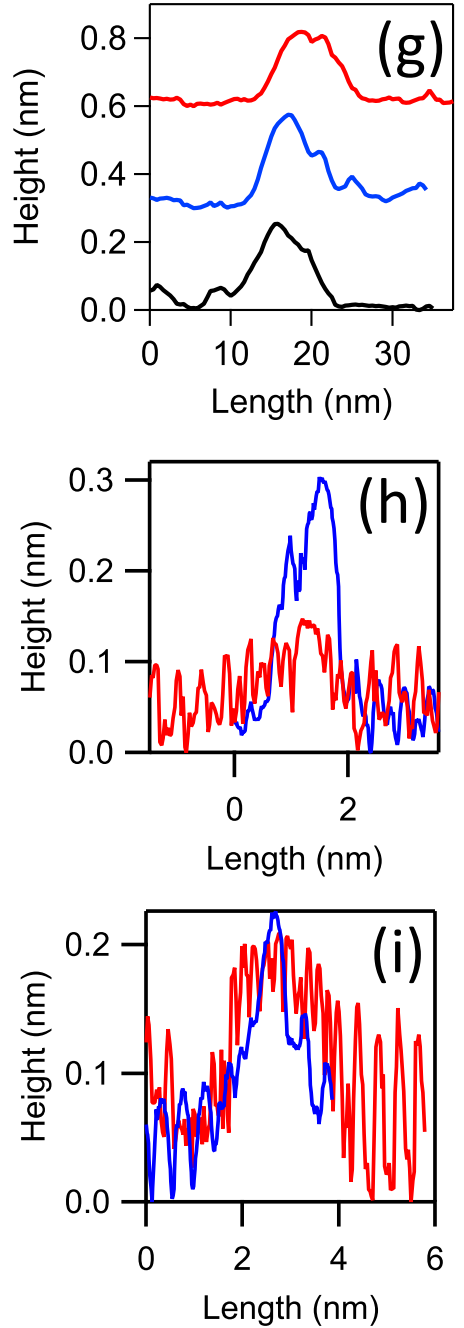

FIG. 1. Examples of defects on graphite induced by ion bombardment. (a)-(d) STM images of clean graphite defects. (e), (f) STM images of Pt-decorated defects. Diamonds in (e), (f) outline the unit cell of the $(\sqrt{3} \times \sqrt{3}) R 30^{\circ}$ superlattice. (g) Line profiles corresponding to lines in STM image (c). (h) Line profiles corresponding to red and blue lines in STM images (a), (e). (i) Line profiles corresponding to red and blue lines in STM images (b,f). $V_{\text {tip }}$ and $I$ are (a) $0.05 \mathrm{~V}, 0.45 \mathrm{nA}$; (b) $0.03 \mathrm{~V}, 0.45 \mathrm{nA}$; (c) $1.2 \mathrm{~V}, 0.26 \mathrm{nA}$; (d) $0.1 \mathrm{~V}, 0.26 \mathrm{nA}$; (e), (f) $0.07 \mathrm{~V}, 0.4 \mathrm{nA}$.

while encapsulated clusters emerge at higher temperature [7-10]. To guide any exploration of surface intercalation by different metals, it is important to gauge the optimal value of $T_{\mathrm{dep}}$, which we define as the point where there is a large population of encapsulated clusters with minimal bare (unencapsulated) clusters adsorbed on the graphite surface. The optimal $T_{\text {dep }}$ values for the three transition metals that we have reported to date- $\mathrm{Cu}, \mathrm{Ru}$, and $\mathrm{Fe}-$ scale quite linearly with the metal's cohesive energy [27], as shown in Fig. 2(a). As explained in Sec. I, we conjecture that this correlation reflects irreversible heterogeneous nucleation of metals at entry portals at low $T_{\text {dep }}$, which blocks the portals.

By fitting the data to a straight line as shown in Fig. 2(a), and using $5.84 \mathrm{eV} /$ atom as the cohesive energy of Pt [27], the optimal $T_{\text {dep }}$ for Pt is predicted to be about $1080 \mathrm{~K}$. Our experimental strategy is to start at a lower temperature, where bare metal clusters can be observed and characterized, then increase $T_{\text {dep }}$ and place a fine grid of $50-100 \mathrm{~K}$ near the value of $T_{\text {dep }}$ where optimal encapsulation is predicted.

\section{Pt growth}

Temperature dependence. $\mathrm{Pt}$ was deposited on graphite at different $T_{\text {dep }}$ as shown in Fig. 3. Focusing on Pt on $i$ graphite first, at $300 \mathrm{~K}$, Pt forms round and small clusters ( $0.65 \pm 0.10 \mathrm{~nm}$ tall, averaged over $X=60$ measurements). At $800 \mathrm{~K}$, much less $\mathrm{Pt}$ is observed on the surface than at $300 \mathrm{~K}$. Some Pt forms tall (bright) clusters on the surface, with heights of $1.14 \pm 0.24 \mathrm{~nm}(X=57)$. The amount of tall clusters continues to decrease at $900 \mathrm{~K}$. At $950 \mathrm{~K}$, however, in addition to the tall clusters, a unique feature appears that has a rounded profile and also a round footprint. Examples are marked by arrows in Figs. 3(c)-3(e). Starting at $1050 \mathrm{~K}$, the number density of the rounded features becomes much smaller, and the tall clusters primarily decorate graphite step edges with only a few on graphite terraces. Finally, at $1100 \mathrm{~K}$, the rounded features are scarce, rendering graphite terraces quite clean. At this high $T_{\text {dep }}$, tall clusters are only found along graphite steps [Fig. 3(f)]; round features are not observed along steps. In the following, we will present evidence that 

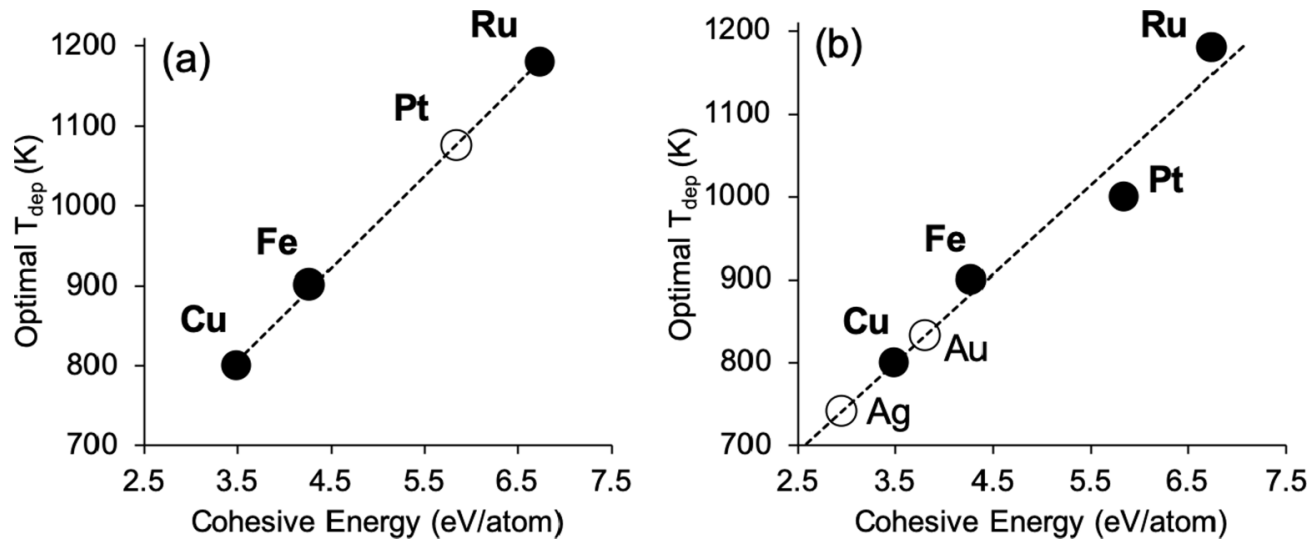

FIG. 2. Plots of optimal $T_{\text {dep }}$ vs cohesive energy. (a) Data points (black circles) for $\mathrm{Cu}, \mathrm{Fe}$, and $\mathrm{Ru}$ (the three transition metals already reported in the literature [8-10]). Open circle shows the prediction for Pt. Dashed line shows linear best fit to the three data points, described by $y=116 x+398$ with a coefficient of determination, $R^{2}=1.00$. (b) Data points (black circles) for $\mathrm{Cu}, \mathrm{Fe}, \mathrm{Ru}$, and $P t$. Open circles show predictions for Ag and Au. Dashed line shows linear best fit to the four data points, described by $y=107 x+424$ and $R^{2}=0.95$.

the tall features are bare clusters, and the rounded features are encapsulated islands. The trends in dimensions and densities of these two types of features, with increasing $T_{\mathrm{dep}}$, are shown in Fig. 4, and are discussed more fully below.

As a control experiment, $\mathrm{Pt}$ was also deposited on $p$ graphite at $1000 \mathrm{~K}$ as shown in Fig. 3(g). Graphite terraces are clean without noticeable features, which contrasts $\mathrm{Pt}$ on $i$-graphite at $1000 \mathrm{~K}$ where rounded features are observed as in Fig. 3(d). This experiment demonstrates that the defects introduced by ion sputtering are essential to produce the rounded features.

Identification of rounded features as encapsulated Pt clusters. To further investigate the rounded features on $i$-graphite at 950-1100 K, we examine high-resolution STM images of rounded features shown in Fig. 5. Zooming in directly on these rounded features, we resolve a hexagonal lattice that has spacing of $0.247 \pm 0.003 \mathrm{~nm}(X=26)$. The spacing corresponds to that of the graphite lattice. Furthermore, the graphite lattice is resolved on both graphite substrate and edges of the rounded features, demonstrating the continuity of the graphite lattice, as shown in Figs. 5( $\left.\mathrm{a}^{\prime}\right)$ and $5\left(\mathrm{~b}^{\prime}\right)$. The robust observation of a continuous graphite overlayer serves to identify these rounded features as encapsulated Pt clusters.

Atop all of the rounded features with resolved graphite lattice, triangular arrays of $\mathrm{C}$ atoms are observed, where only three out of six $\mathrm{C}$ atoms are visible in a honeycomb. As discussed elsewhere, this threefold symmetry can signal a single graphene sheet interacting strongly with a metal substrate, known to occur for $\mathrm{Ru}(0001)$ [36-39]; or multiple graphene sheets stacked in a way that breaks the sixfold symmetry, e.g., the conventional AB stacking of graphite [24]. At present the appropriate interpretation for $\mathrm{Pt}$ is not clear. We note that precedent exists for both interpretations: multiple graphene sheets have been observed atop metal clusters for two other
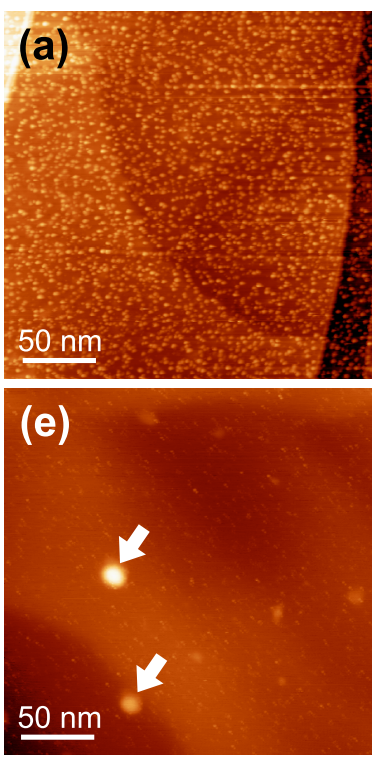
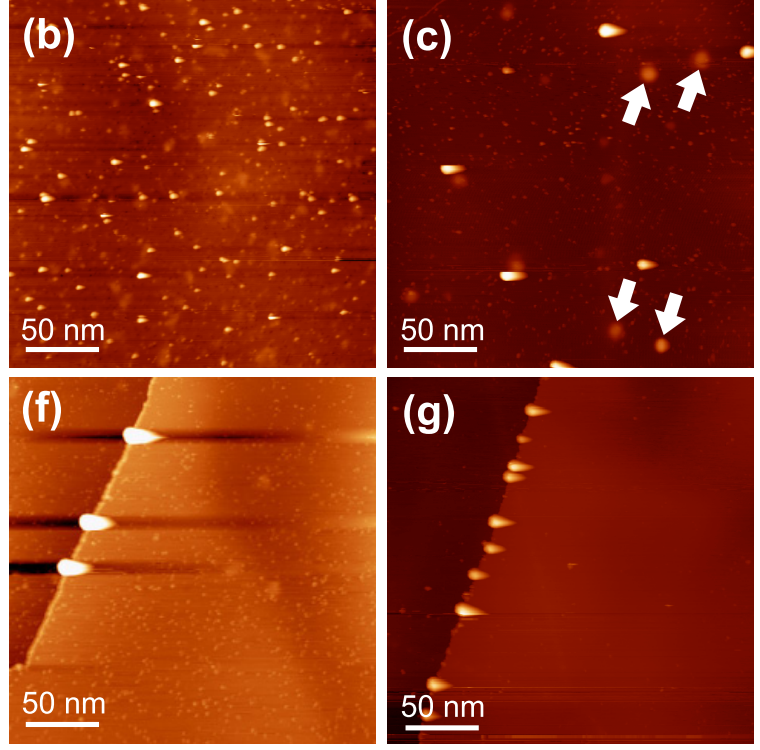

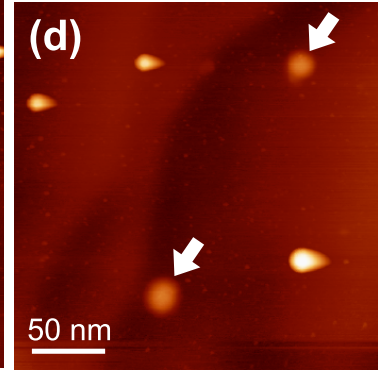

FIG. 3. STM images after seven separate depositions of Pt on $i$-graphite at (a) $300 \mathrm{~K}$, (b) $800 \mathrm{~K}$, (c) $950 \mathrm{~K}$, (d) $1000 \mathrm{~K}$, (e) $1050 \mathrm{~K}$, and (f) $1100 \mathrm{~K}$, and on $p$-graphite at $(\mathrm{g}) 1000 \mathrm{~K}$. Examples of rounded features are marked by arrows. Tip bias $\left(V_{\text {tip }}\right)$ and tunneling current $(I)$ are (a) $4.0 \mathrm{~V}, 0.28 \mathrm{nA}$; (b) $4.5 \mathrm{~V}, 0.27 \mathrm{nA}$; (c) $1.2 \mathrm{~V}, 0.27 \mathrm{nA}$; (d) $1.6 \mathrm{~V}, 0.27 \mathrm{nA}$; (e) $1.3 \mathrm{~V}, 0.26 \mathrm{nA}$; (f) $1.1 \mathrm{~V}, 0.26 \mathrm{nA}$; (g) $2.7 \mathrm{~V}, 0.27 \mathrm{nA}$. 

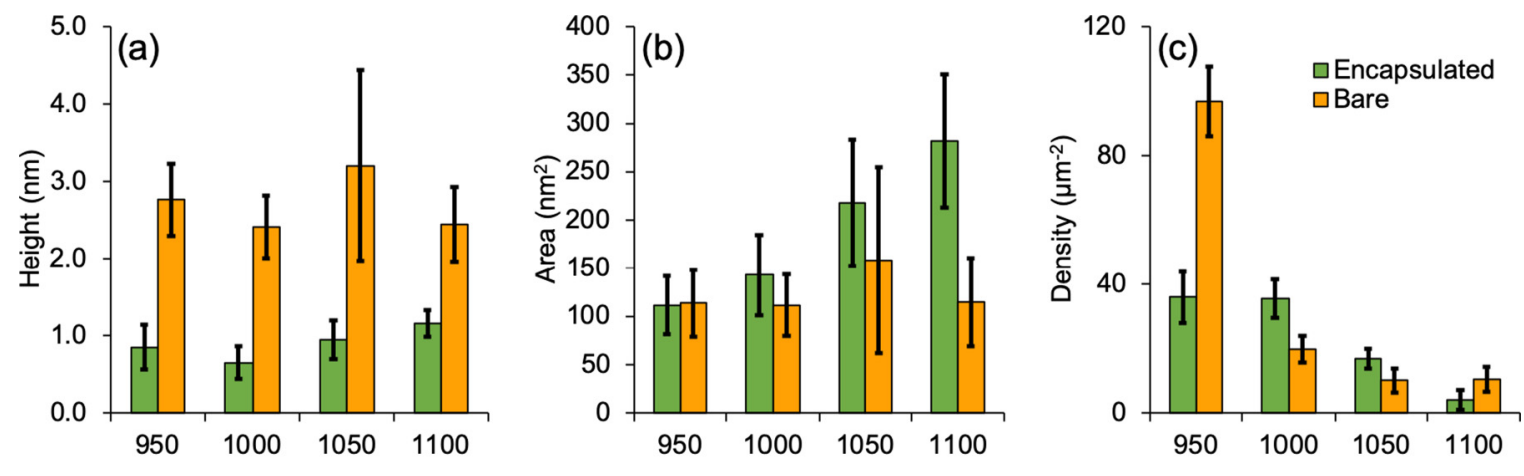

Deposition Temperature $(\mathrm{K})$

FIG. 4. Average (a) height, (b) area, and (c) density of Pt clusters: Encapsulated cluster (green bars) and bare clusters (gold bars). Values for individual data points are given in Table S1 in the Supplemental Material [20]. All measurements are for features on terraces only.

metals, $\mathrm{Cu}[8]$ and $\mathrm{Fe}[10]$, whereas a single graphene sheet has been identified atop $\mathrm{Ru}[9]$.

In general, there are noticeable defectlike features on top of and beside encapsulated Pt clusters. These features are espe- cially visible in Fig. 5. More examples of atomically resolved defectlike features are shown in Figs. 1(e) and 1(f). These features are similar, whether they are on encapsulated clusters or beside them on the graphite substrate. Many of these
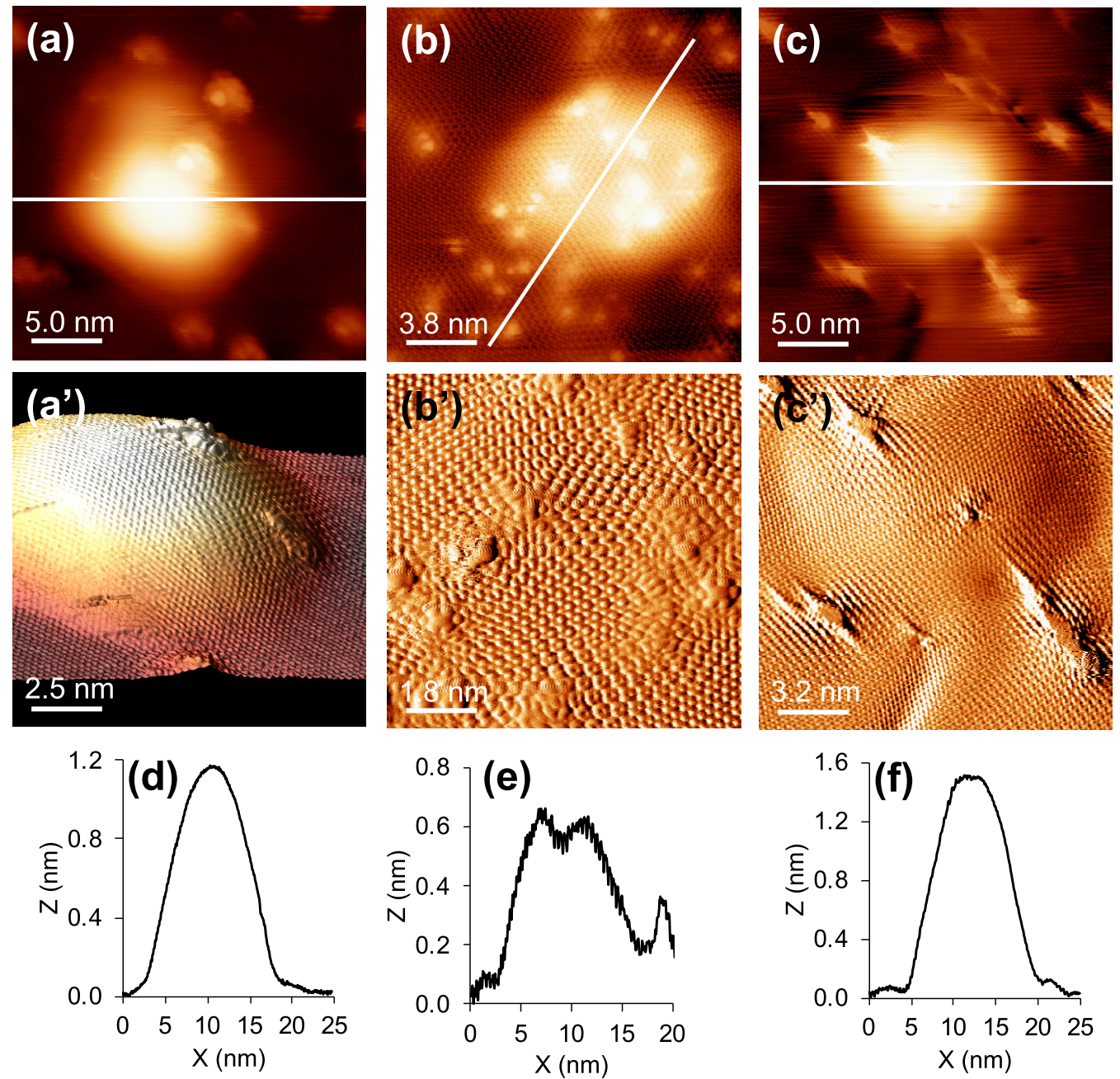

FIG. 5. High-resolution STM images of encapsulated Pt clusters at (a), (a') $950 \mathrm{~K}$, (b), (b') $1000 \mathrm{~K}$, and (c), (c') $1050 \mathrm{~K}$. The continuity of graphite lattice is seen in $\left(\mathrm{a}^{\prime}\right)$ a three-dimensional image and $\left(\mathrm{c}^{\prime}\right)$ a derivative image. $\left(\mathrm{b}^{\prime}\right)$ is a derivative image zoomed in directly on top of the island in (b). Corresponding line profiles are shown in (d)-(f). $V_{\text {tip }}$ and $I$ are (a) $0.07 \mathrm{~V}, 0.41 \mathrm{nA}$; (a') $0.006 \mathrm{~V}, 0.41 \mathrm{nA}$; (b), (b') $0.07 \mathrm{~V}^{\prime}, 0.40$ $\mathrm{nA}$; (c) $0.08 \mathrm{~V}, 0.41 \mathrm{nA}$; (c') $0.07 \mathrm{~V}, 0.41 \mathrm{nA}$. 

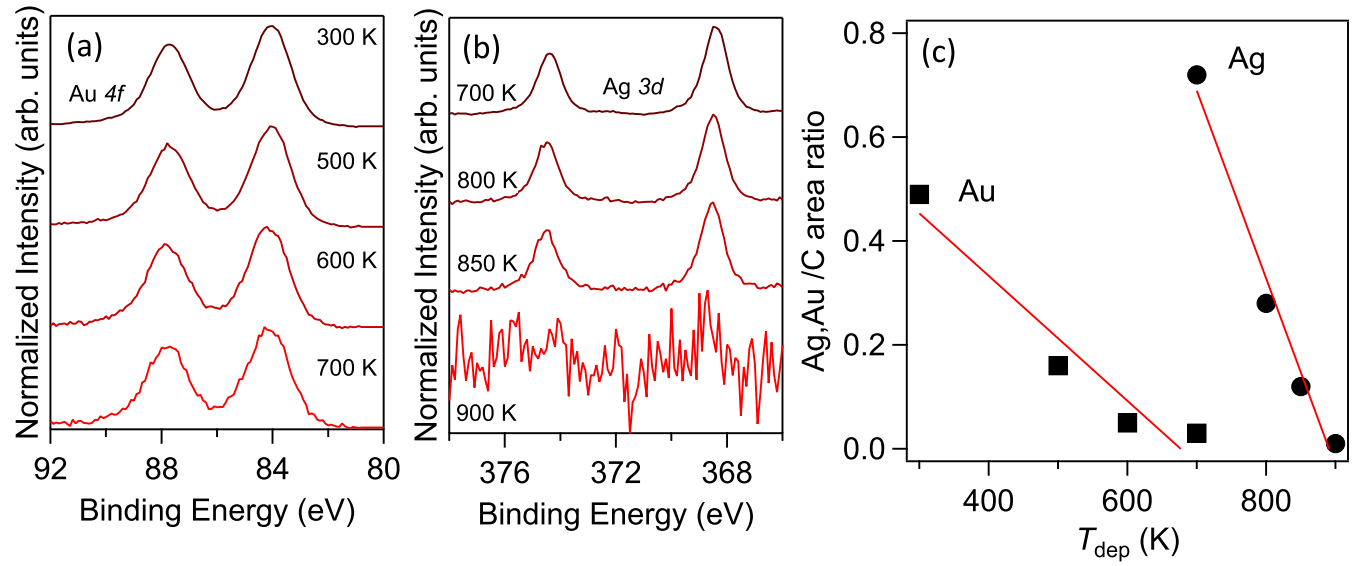

FIG. 6. Nomalized XPS spectra of Au $4 f$ (a) and Ag 3d (b) after Au and Ag depositions on $i$-graphite at varrious $T_{\text {dep. }}$ (c) Peak area ratios of metal:carbon XPS peaks, as functions of $T_{\text {dep }}$. Circles: Ag $3 d_{5 / 2}$ to $\mathrm{C} 1 s$ ratio as a function of $T_{\text {dep }}$ for Ag deposited on $i$-graphite. Red line represents linear best fit with $R^{2}=0.980$. Numerical values of $\mathrm{Ag} / \mathrm{C}$ from the lowest to highest $T_{\text {dep }}$ are $0.72,0.28,0.12$, and 0.01 , respectively. Squares: $\mathrm{Au} 4 f_{7 / 2}$ to $\mathrm{C} 1 \mathrm{~s}$ ratio as a function of $T_{\mathrm{dep}}$ for Au deposited on $i$-graphite. Red line denotes linear best fit with $R^{2}=0.932$. Numerical values for data points from the lowest to highest $T_{\text {dep }}$ are $0.49,0.16,0.05$, and 0.03 .

features have a somewhat compact, corpuscular appearance, which distinguishes them from the diffuse appearance of preexisting defects that result from ion bombardment alone. They are usually slightly taller than preexisting defects [Fig. 1(h)]. Thus, it is reasonable to assign features like the brightest ones in Figs. 1(e) and 1(f) as a few Pt atoms anchored at defect sites. Elsewhere, we have made similar arguments to identify $\mathrm{Cu}$-decorated defects [8].

Multiple moiré patterns have been reported on epitaxial graphene grown on $\mathrm{Pt}(111)$ [40,41]. However, no moiré is observed on encapsulated Pt clusters in the present study. The absence of moiré is potentially due to defects that interfere with the undulation of the $\mathrm{C}$ overlayer. Another possibility is that multiple graphene layers exist atop the Pt island, which would also dampen or extinguish the moiré, as they do on $\mathrm{Ru}(0001)$ [36].

In keeping with literature reports of low reactivity between Pt and C [42,43], there is no known form of Pt carbide. Similar to $\mathrm{Ru}$, the solubility of solid $\mathrm{C}$ in solid $\mathrm{Pt}$ is low, at only 1.76 at. $\%$ at $1270 \mathrm{~K}$. Thus, it is highly likely that the encapsulated Pt clusters are metallic in nature, as are other encapsulated transition metals [8-10].

Encapsulated Pt cluster densities and dimensions. Having identified the rounded features as encapsulated metallic Pt clusters, we comment on their relative density, which is shown in Fig. 4(c). At $950 \mathrm{~K}$, the density of bare islands is much higher than that of encapsulated Pt clusters. At slightly higher temperatures of 1000 and $1050 \mathrm{~K}$, the relationship is reversed, with encapsulated clusters outnumbering bare clusters. At $1000 \mathrm{~K}$ the absolute density of encapsulated clusters is about twice that at $1050 \mathrm{~K}$. Together, these observations lead us to conclude that the optimal $T_{\text {dep }}$ for $\mathrm{Pt}$, under our experimental conditions, is $1000 \mathrm{~K}$, only $80 \mathrm{~K}$ below the value predicted from cohesive energies (Sec. III B).

We also measure the cluster dimensions as a function of $T_{\text {dep }}$ as shown in Figs. 4(a) and 4(b). The average encapsulated cluster height increases with deposition temperature, especially from 1000 to $1100 \mathrm{~K}$. Cluster area increases smoothly throughout the temperature range. Thus, an increase in cluster dimensions accompanies a decrease in island density as $T_{\mathrm{dep}}$ increases.

\section{Ag growth}

Temperature dependence for Ag: Predictions. Following the same line of reasoning as presented in Sec. III B for Pt, the predicted optimal $T_{\text {dep }}$ for $\mathrm{Ag}$ is about $740 \mathrm{~K}$, based on its cohesive energy of $2.95 \mathrm{eV} /$ atom. This is shown by the open circle labeled Ag in Fig. 2(b), where the experimental value for Pt has been added to the dataset used to make the prediction. To also help guide in the search, we measured Ag $3 d$ peak intensity (which should at least roughly correlate with Ag coverage) and binding energy as a function of $T_{\text {dep }}$, with results shown in Figs. 6(a) and 6(c). The peak intensity is measurable through $850 \mathrm{~K}$, but reaches zero at $900 \mathrm{~K}$, indicating that $850 \mathrm{~K}$ is the practical upper limit. The binding energy of the Ag $3 d$ peak is constant at $368.5 \mathrm{eV}$, consistent with metallic Ag. We thus searched for Ag encapsulation in the range $700-850 \mathrm{~K}$.

STM results, 700-850 K. Starting our examination at $700 \mathrm{~K}$, Figs. 7(a)-7(d) show representative STM images. As observed for $\mathrm{Cu}$ [8], bare features are easily removed from the graphite surface during scanning. For example, the feature present in Fig. 7(a) is gone in several subsequent images, one of which is shown in Fig. 7(b). Locations of surrounding features are circled as reference points to guide the eye. After removal of bare Ag, the STM tip usually becomes unstable, which can be remedied by pulsing the tip between a large voltage difference, for example, -10 to $+10 \mathrm{~V}$. There are also other bare Ag features [Fig. 7(c) and 7(d)] but they are all removed by the STM tip during scanning, even though they can be quite large in size, as shown by the profiles in Figs. 7(e) and 7(f).

At $800 \mathrm{~K}, \mathrm{Ag}$ deposited on $i$-graphite generally results in graphite step edges decorated by bare clusters and relatively empty graphite terraces as seen in Fig. 7(g). Small bare clusters on terraces are about 1.0-1.2 nm tall, as shown in Figs. 7(h) and 7(k). Some of the bare clusters are unstable under tunneling, so they can be removed by the STM tip, resulting in minor streaking in scanning as pointed by an 

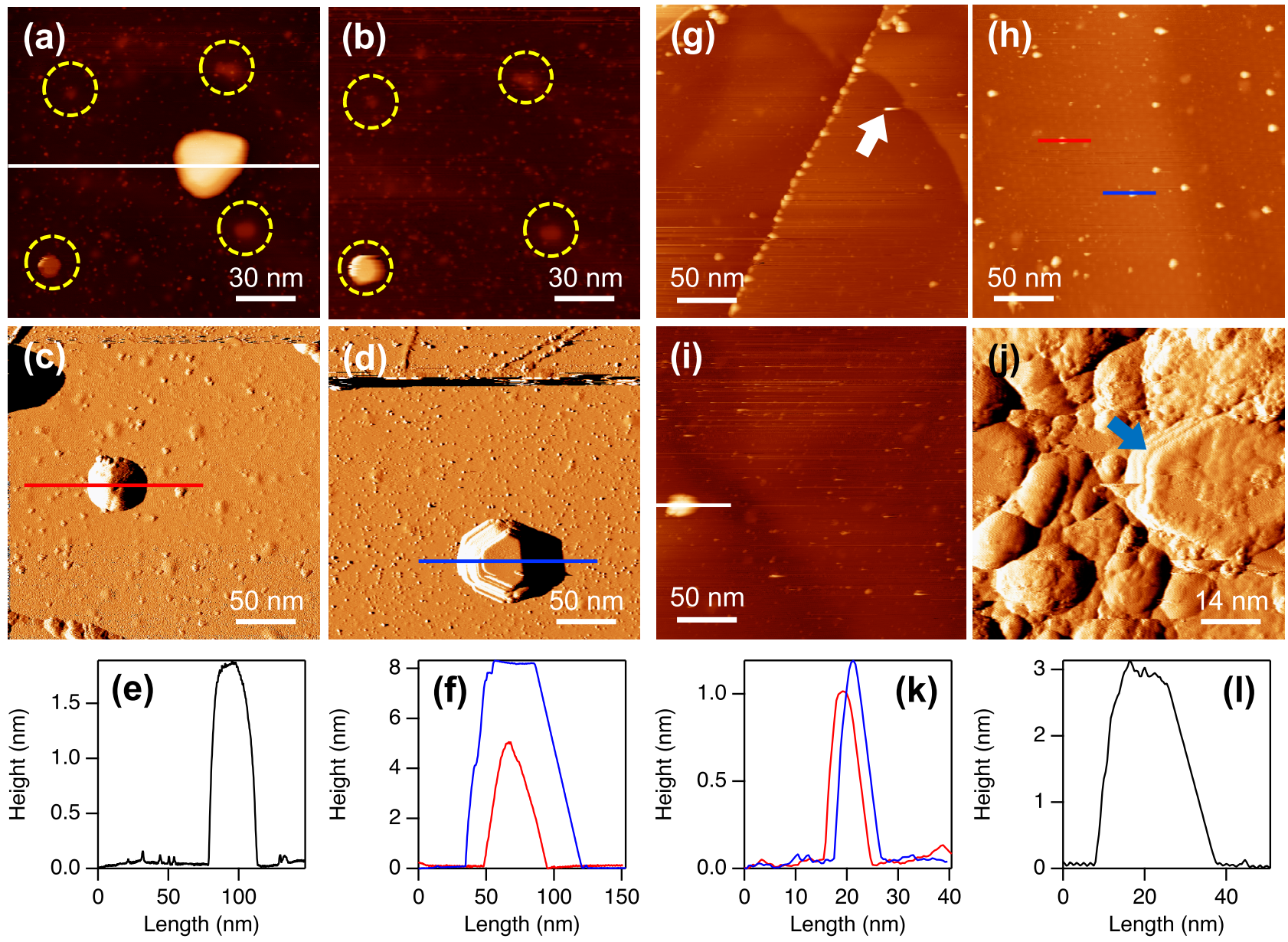

FIG. 7. Representative STM images and select line profiles, for Ag on $i$-graphite at various $T_{\text {dep }}$. (a) - (d) $T_{\text {dep }}=700 \mathrm{~K}$, and (g)-(j) $T_{\text {dep }}=$ $800 \mathrm{~K}$. Bare Ag feature in (a) is removed in subsequent image (b). Arrow in (g) points out streaking in a bare cluster. Arrow in (j) points to a bare Ag island that is subsequently removed by the STM tip, along with most surrounding features. Line profile in (e) corresponds to white horizontal line in image (a), and blue/red profiles in (f) correspond to blue/red lines in images (c), (d). Blue/red line profiles in (k) correspond to blue/red horizontal lines in (h) and the profile in (l) corresponds to the white line in (i). (a), (b), (g-i) are topographic and (c), (d), (j) are derivative STM images. $V_{\text {tip }}$ and $I$ are (a) $1.2 \mathrm{~V}, 0.27 \mathrm{nA}$; (b) $0.8 \mathrm{~V}, 0.28 \mathrm{nA}$; (c) $1.3 \mathrm{~V}, 0.27 \mathrm{nA}$; (d) $2.4 \mathrm{~V}, 0.27 \mathrm{nA}$; (g) $1.7 \mathrm{~V}, 0.26 \mathrm{nA}$; (h) 1.4 $\mathrm{V}, 0.26 \mathrm{nA}$; (i) $2.0 \mathrm{~V}, 0.27 \mathrm{nA}$; (j) $2.6 \mathrm{~V}, 0.27 \mathrm{nA}$.

arrow in Fig. 7(g). This behavior is similar to $\mathrm{Cu}$ deposited on $i$-graphite and $p$-graphite at $300 \mathrm{~K}$, where bare $\mathrm{Cu}$ clusters are unstable during scanning and can be picked up by the STM tip [8].

Rarely, there is an area that has a significantly higher coverage of bare Ag than elsewhere on graphite as shown in Fig. 7(j). An arrow points out a feature with a rough top and hexagonal footprint. In the process of zooming in on this feature, tunneling becomes unstable, and the hexagonal feature along with surrounding lumps are removed by the tip. In this case, the removal of bare Ag likely results from reducing the bias voltage during zooming in, which leads to a smaller tip-sample distance and causes the tip to interact more strongly with the Ag. This indicates that the hexagonal feature is a bare Ag island that is unstable under tunneling, similar to the large clusters in Figs. 7(a)-7(d).

After Ag deposition at $850 \mathrm{~K}$, the graphite terraces are almost cluster free, even cleaner than at $800 \mathrm{~K}$. Terraces show defects resembling the clean ones in Figs. 1(a) and 1(b), indicating that the defects no longer trap Ag. Very rarely, a larger feature is observed on a terrace, but it exhibits the streaking characteristic of a bare cluster.

Overall, the above observations of instability during tunneling suggest that the clusters observed after Ag deposition at $700-850 \mathrm{~K}$ are bare clusters. In contrast, encapsulated metal clusters are always very stable under tunneling, based on data for $\mathrm{Cu}, \mathrm{Fe}, \mathrm{Ru}$, and $\mathrm{Pt}$. For $\mathrm{Ag}$, we find no evidence of stable, encapsulated metal clusters.

\section{E. Au growth}

Temperature dependence for Au: Predictions. Following the same line of reasoning as presented in Sec. III B for $\mathrm{Pt}$ or Sec. III D for $\mathrm{Ag}$, the predicted optimal $T_{\mathrm{dep}}$ for $\mathrm{Au}$ is about $830 \mathrm{~K}$, based on its cohesive energy of $3.81 \mathrm{eV} /$ atom [see Fig. 2(b)]. However, from XPS, Au coverage approaches zero at about $700 \mathrm{~K}$ [Figs. 6(b) and 6(c)]. Also similar to Ag, the binding energy of the $\mathrm{Au} 4 f$ peak is constant at $84.1 \mathrm{eV}$, consistent with metallic Au. This suggests that it may be impractical to reach conditions where Au becomes encapsulated, 

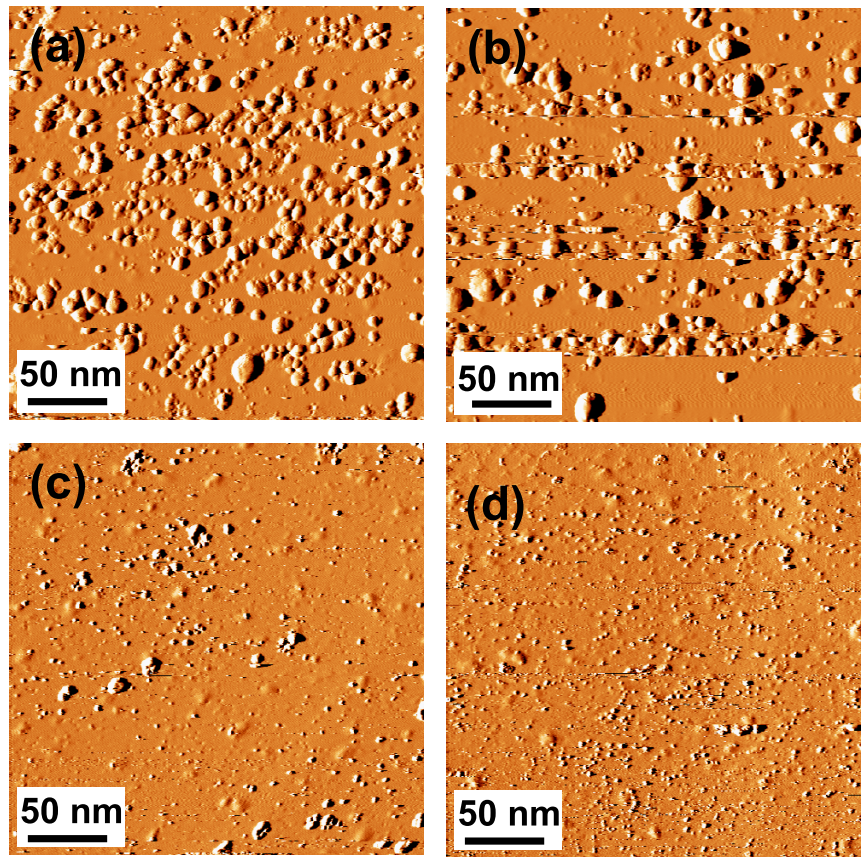

FIG. 8. Representative STM images of Au deposited on $i$ graphite at (a) $300 \mathrm{~K}$ (b) $500 \mathrm{~K}$, (c) $600 \mathrm{~K}$, and (d) $700 \mathrm{~K}$. All images are derivative STM images. $V_{\text {tip }}$ and $I$ are (a) $4.8 \mathrm{~V}, 0.26 \mathrm{nA}$; (b) $4.9 \mathrm{~V}$, $0.26 \mathrm{nA}$; (c) $4.6 \mathrm{~V}, 0.28 \mathrm{nA}$; (d) $4.1 \mathrm{~V}, 0.26 \mathrm{nA}$.

under our experimental conditions. We nonetheless searched for Au encapsulation in the range $300-700 \mathrm{~K}$.

STM results, 300-700 K. As shown in Fig. 8(a), Au deposited on $i$-graphite at $300 \mathrm{~K}$ forms bare clusters that are 1-3 nm tall. Similar to $\mathrm{Ag}$ and $\mathrm{Cu}$ on $i$-graphite, bare $\mathrm{Au}$ clusters can be perturbed by the STM tip during scanning, causing frequent streakiness and image instabilities. To reduce or prevent streaking, bias voltage was usually kept high $(>4.0 \mathrm{~V})$ during scanning.

Additional depositions of Au on $i$-graphite above $300 \mathrm{~K}$, in the range $T_{\text {dep }}=500-700 \mathrm{~K}$, result in decreasing coverages of Au on the graphite surface, evident in Figs. 8(b)-8(d) and the downward trend in Fig. 6(c). There is no evidence of encapsulated Au clusters. In order to broaden our parameter space somewhat, we performed two other tests: (i) increasing defect density in graphite by increasing ion emission current during ion bombardment, prior to Au deposition; and (ii) depositing $\mathrm{Au}$ at $300 \mathrm{~K}$, then annealing to $700 \mathrm{~K}$. In both cases the only result was bare Au clusters on terraces, with STM images similar to those in Figs. 8(a)-8(d). There was no evidence of encapsulated Au clusters.

In summary, Ag and Au do not form encapsulated clusters at the graphite surface, whereas Pt does. We next examine whether this is consistent with theoretical predictions.

\section{DFT CALCULATIONS}

$P t \pm$ graphite. We first examine the adsorption energies of one Pt adatom after relaxation from the seven high-symmetry positions on graphite: TH, TT, HT, BM, MB, MM, and CC, as shown in Fig. 9(a). As shown in Table I, the most favorable adsorption (top) site is at BM with adsorption energy $-1.813 \mathrm{eV}$. The adsorption of a Pt adatom at MB, CC, and MM sites
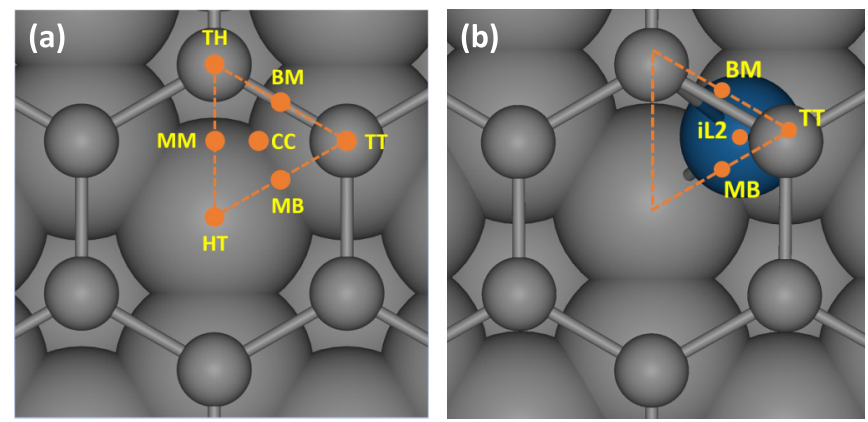

FIG. 9. (a) Top view of seven initial positions (TH, TT, HT, BM, $\mathrm{MB}, \mathrm{MM}$, and $\mathrm{CC}$ ) of a metal atom with respect to the graphite substrate for DFT calculations. The letters T, H, B, M, and C stand for top, hollow, bridge, midpoint, and center, respectively. The first letter denotes location with respect to the top GML, and the second letter denotes location with reference to the second GML from the top. (b) Top view of the most favorable configuration for one Pt atom (blue) intercalated underneath the top GML (small gray balls) from DFT calculations. The site close to the midpoint between the initial sites iMB and iTT is designated iL2.

is unstable, and the adatom will move to a local minimum close to the initial site after full relaxation. Thus, the minimum energy path for diffusion of the adatom should be along TT to $\mathrm{BM}$ to $\mathrm{TH}$, and the lower limit of the diffusion barrier is about

TABLE I. DFT results for chemical potential $\mu_{\mathrm{Pt}}$ (in units of $\mathrm{eV}$ ) and magnetic moment $m$ (in units of Bohr magneton per cell) for adsorption and intercalation of one Pt atom after full relaxation at different initial sites ("t" denotes adsorption on top of graphite, and "i" denotes intercalation beneath the top GML). Sites BM, CC, etc., are defined in Fig. 9(a) and its caption. $\mu_{\mathrm{Pt}}$ is calculated from Eq. (1). $\Delta \mu_{\mathrm{Pt}}$ is the energy (or chemical potential) difference relative to the lowest-energy adsorption site, tBM.

\begin{tabular}{|c|c|c|c|c|}
\hline Site & $\mu_{\mathrm{Pt}}$ & $\Delta \mu_{\mathrm{Pt}}$ & $m$ & Notes \\
\hline $\begin{array}{l}\text { tBM } \\
\text { tCC }\end{array}$ & -1.813 & 0.000 & 0.000 & $\begin{array}{l}\text { Local equilibrium } \\
\text { Moves to tBM }\end{array}$ \\
\hline $\begin{array}{l}\text { tHT } \\
\text { tMB } \\
\text { tMM }\end{array}$ & -1.094 & +0.719 & 0.039 & $\begin{array}{l}\text { Local equilibrium } \\
\text { Moves to tTT } \\
\text { Moves to tBM }\end{array}$ \\
\hline $\mathrm{tTH}$ & -1.669 & +0.144 & 0.000 & Local equilibrium \\
\hline $\mathrm{tTT}$ & -1.652 & +0.161 & 0.000 & Local equilibrium \\
\hline iBM & & & & Moves to iL1 ${ }^{\mathrm{a}}$ \\
\hline $\mathrm{iCC}$ & & & & Moves to iL2 ${ }^{\mathrm{b}}$ \\
\hline iHT & & & & Moves to iL $2^{\mathrm{b}}$ \\
\hline iMB & & & & Moves to iL2 ${ }^{\mathrm{b}}$ \\
\hline iMM & & & & Moves to iL3 ${ }^{c}$ \\
\hline iTH & & & & Moves to iL $2^{\mathrm{b}}$ \\
\hline iTT & & & & Moves to iL $2^{\mathrm{b}}$ \\
\hline $\mathrm{iL1}^{\mathrm{a}}$ & -2.035 & -0.222 & 0.000 & Local equilibrium \\
\hline $\mathrm{iL}^{\mathrm{b}}$ & -2.045 & -0.232 & 0.000 & Local equilibrium \\
\hline $\mathrm{iL}^{\mathrm{c}}$ & -2.037 & -0.224 & 0.001 & Local equilibrium \\
\hline
\end{tabular}

${ }^{a}$ iL1 is a local minimum at midpoint between iBM and iTT.

${ }^{b} \mathrm{iL} 2$ is a local minimum (lowest) close to midpoint between iMB and iTT [see Fig. 9(b)].

${ }^{c}$ iL3 is a local minimum close to iCC, with a small shift of top GML. 
TABLE II. DFT results for chemical potential $\mu_{\mathrm{Ag}}$ (in units of $\mathrm{eV}$ ) and magnetic moment $m$ (in units of Bohr magneton per cell) for adsorption and intercalation of one $\mathrm{Ag}$ atom after full relaxation at different initial sites (" $t$ " denotes adsorption on top of graphite, and "i" denotes intercalation beneath the top GML). Sites BM, CC, etc., are defined in Fig. 9(a) and its caption. $\mu_{\mathrm{Ag}}$ is calculated from Eq. (1). $\Delta \mu_{\mathrm{Ag}}$ is the energy (or chemical potential) difference relative to the lowest-energy adsorption site, tTH.

\begin{tabular}{|c|c|c|c|c|}
\hline Initial site & $\mu_{\mathrm{Ag}}$ & $\Delta \mu_{\mathrm{Ag}}$ & $m$ & Notes \\
\hline $\mathrm{tBM}$ & -0.281 & +0.003 & 0.993 & Local equilibrium \\
\hline $\mathrm{tCC}$ & -0.280 & +0.004 & 0.996 & Local equilibrium \\
\hline tHT & -0.272 & +0.012 & 1.001 & Local equilibrium \\
\hline tMB & -0.278 & +0.005 & 1.001 & Local equilibrium \\
\hline tMM & & & & Moves to tTH \\
\hline tTH & -0.283 & 0.000 & 0.990 & Local equilibrium \\
\hline $\mathrm{tTT}$ & -0.282 & +0.001 & 1.003 & Local equilibrium \\
\hline iBM & & & & Moves to iTT \\
\hline $\mathrm{iCC}$ & & & & Moves to iTT \\
\hline iHT & 0.578 & +0.861 & 0.000 & Local equilibrium \\
\hline iMB & & & & Moves to iTT \\
\hline iMM & 0.542 & +0.826 & 0.000 & Local equilibrium \\
\hline iTH & & & & Moves to iTT \\
\hline iTT & 0.515 & +0.798 & 0.000 & Local equilibrium \\
\hline
\end{tabular}

$0.14 \mathrm{eV}$ [the true value would require a nudged elastic band (NEB) calculation].

We also look at the energies of one Pt atom intercalated beneath the top GML. Any intercalated (i) site is more favorable than any top (t) site (Table I), and the most favorable i site is close to the midpoint between iMB and iTT, which is shown in Fig. 9(b). We designate it iL2, and its chemical potential of $-2.045 \mathrm{eV}$ is $0.232 \mathrm{eV}$ lower than the adsorption energy at tBM. Therefore, for one Pt atom, intercalation beneath the top GML is always more favorable than adsorption on top of the graphite surface. We also note that both the adsorption system and the intercalated system have zero or nearly zero magnetism (Table I).

$A g+$ graphite. Let us next examine the adsorption energies of one Ag adatom after relaxation from the same seven initial positions shown in Fig. 9(a). Although the lowest adsorption energy of $-0.283 \mathrm{eV}$ is at the tTH site, all local energy minima for adsorption are very close. The adsorption energies at tTT and tHT are -0.282 and $-0.272 \mathrm{eV}$, respectively. Turning to the energies of one $\mathrm{Ag}$ atom intercalated underneath the top GML, all i sites are less favorable than t sites (Table II). The most favorable i site is at iTT with chemical potential of $+0.515 \mathrm{eV}$, which is $0.789 \mathrm{eV}$ higher than the adsorption energy at $\mathrm{tTH}$. Therefore, for one $\mathrm{Ag}$ atom, intercalation beneath the top GML is always less favorable than adsorption on top of the graphite surface. As an aside, the adsorption system is magnetic and becomes nonmagnetic after intercalation (Table II). (This feature is very similar to $\mathrm{Cu}$ adsorption and intercalation at another layered material, $\mathrm{MoS}_{2}$ [44].)

$A u+$ graphite. Finally, we look at the adsorption energies of one $\mathrm{Au}$ adatom after relaxation from the seven initial positions. The lowest adsorption energy $-0.492 \mathrm{eV}$ is at the tTH site, which is the same as that for Ag. For one Au atom intercalated beneath the top GML, the only local stable site
TABLE III. DFT results for chemical potential $\mu_{\mathrm{Au}}$ (in units of $\mathrm{eV}$ ) and magnetic moment $m$ (in units of Bohr magneton per cell) for adsorption and intercalation of one Au atom after full relaxation at different initial sites ("t" denotes adsorption on top of graphite, and "i" denotes intercalation beneath the top GML). Sites BM, CC, etc., are defined in Fig. 9(a) and its caption. $\mu_{\mathrm{Au}}$ is calculated from Eq. (1). $\Delta \mu_{\mathrm{Au}}$ is the energy (or chemical potential) difference relative to the lowest-energy adsorption site, $\mathrm{tTH}$.

\begin{tabular}{|c|c|c|c|c|}
\hline Initial site & $\mu_{\mathrm{Au}}$ & $\Delta \mu_{\mathrm{Au}}$ & $m$ & Notes \\
\hline $\mathrm{tBM}$ & & & & Moves to $\mathrm{tTH}$ \\
\hline $\mathrm{tCC}$ & & & & Moves to tTH \\
\hline tHT & -0.434 & +0.059 & 0.779 & Local equilibrium \\
\hline tMB & & & & Moves to tTT \\
\hline tMM & & & & Moves to $\mathrm{tTH}$ \\
\hline $\mathrm{tTH}$ & -0.492 & 0.000 & 0.947 & Local equilibrium \\
\hline $\mathrm{tTT}$ & -0.484 & +0.008 & 0.925 & Local equilibrium \\
\hline iBM & & & & Moves to iTT \\
\hline $\mathrm{iCC}$ & & & & Moves to iTT \\
\hline $\mathrm{iHT}$ & & & & Moves to iTT \\
\hline iMB & & & & Moves to iTT \\
\hline iMM & & & & Moves to iTT \\
\hline iTH & & & & Moves to iTT \\
\hline iTT & 0.735 & +1.227 & 0.000 & Local equilibrium \\
\hline
\end{tabular}

is iTT (again the same as for Ag). It has $\mu_{M}=+0.735 \mathrm{eV}$, which is $1.227 \mathrm{eV}$ higher than at $\mathrm{tTH}$. Therefore, for one $\mathrm{Au}$ atom, intercalation beneath the top GML is always less favorable than adsorption on top of the graphite surface. Also, the adsorption system is magnetic while the encapsulated system is nonmagnetic (Table III). (Again, this feature is very similar to $\mathrm{Cu}$ adsorption and intercalation on $\mathrm{MoS}_{2}$ [44].)

In summary, the DFT results for single metal atoms predict that intercalation is favorable for $\mathrm{Pt}$ but unfavorable for $\mathrm{Ag}$ and $\mathrm{Au}$, in agreement with the experimental results for surface encapsulation.

\section{DISCUSSION}

The main result of this paper is the experimental observation and theoretical prediction that $\mathrm{Pt}$ metal clusters can become encapsulated at the surface of graphite, whereas $\mathrm{Ag}$ and Au cannot. These transition metals, unlike others, have been found to not undergo encapsulation. Counterexamples include not only $\mathrm{Pt}$, but also $\mathrm{Cu}, \mathrm{Ru}$, and $\mathrm{Fe}$, as reported elsewhere [8-10].

This result supports the validity of using single-metal-atom energetics to predict encapsulation. As noted in Sec. I, we have postulated that the key to encapsulation is having a high population of single atoms beneath the top GML (a high-density two-dimensional gas), so that there is a high probability of nucleation and growth, leading to irreversible trapping. For each of the other transition metals, $\mathrm{Cu}, \mathrm{Ru}$, and $\mathrm{Fe}$, we have reported that single-metal-atom energetics favor encapsulation, even in the case of $\mathrm{Cu}$, where the stability of embedded $\mathrm{Cu}$ clusters or embedded $\mathrm{Cu}$ slabs predicts the opposite [8]. In the present study, encapsulation of $\mathrm{Ag}$ and Au single atoms is strongly disfavored-by 0.8 and $1.2 \mathrm{eV}$ relative to adsorption on top of graphite, whereas encapsulation of Pt is favored by about $0.2 \mathrm{eV}$. The exact reason why 
$\mathrm{Ag}$ and $\mathrm{Au}$ are so different (even from $\mathrm{Cu}$ ), in terms of their interaction with graphite, is a topic of current investigation in our group. The validity of using single-metal-atom (plus graphite) calculations to predict encapsulation is an important result, because calculations involving single metal atoms are generally simpler and less expensive than calculations involving metal clusters or metal slabs (plus graphite).

Elsewhere, we have argued that encapsulated metal islands tend to fall into two groups: Those with small sizes and high number densities (Ru, Dy) and those with large sizes and low densities $(\mathrm{Cu}, \mathrm{Fe})$ [10]. We speculated that this might reflect a division between clusters that diffuse and ripen $(\mathrm{Cu}$, $\mathrm{Fe})$ vs those that are fixed in place and do not ripen ( $\mathrm{Ru}$, Dy). Pt appears to be intermediate in its properties. The Pt island heights around $1 \mathrm{~nm}$, and widths around $10 \mathrm{~nm}$, resemble those of $\mathrm{Ru}$ and Dy [10]. However, the rather low maximum density of $36 \mu \mathrm{m}^{-2}$ resembles that of $\mathrm{Cu}$ and $\mathrm{Fe}$ [10]. Perhaps in this case the low density has a different origin than diffusion-mediated coarsening. For Pt, the difference in $\mu_{M}$ between the most favored adsorption site and the most favored interstitial site is only $0.2 \mathrm{eV}$. This is rather small when compared with the other three transition metals, for which the difference in $\mu_{M}$ ranges from $0.5 \mathrm{eV}$ for $\mathrm{Cu}$ to $1.2 \mathrm{eV}$ for Fe. Hence the density of the single-Pt-atom gas beneath the graphite surface is relatively low, leading to a lower probability for nucleation and growth. We thus propose that the density of Pt islands is low because the probability of nucleation and growth is low.

The shapes of the Pt islands are also distinctive. All other encapsulated metal islands investigated until now have flat tops, though for $\mathrm{Cu}$ round-top islands can be observed in addition to flat-top islands, depending on island size [8]. In other systems the islands with flat tops also show faceted footprints, which are absent for Pt. The factors that favor round vs flat tops, and correspondingly round vs faceted footprints, are not presently understood.

\section{CONCLUSIONS}

Using STM, we have shown that Pt clusters can be embedded beneath the surface of graphite, whereas $\mathrm{Ag}$ and
Au cannot. This is in complete agreement with independent predictions from density functional theory, which show that surface intercalation of single metal atoms is favorable for $\mathrm{Pt}$, but unfavorable for $\mathrm{Ag}$ and $\mathrm{Au}$. This agreement between theory and experiment supports the validity of using singlemetal-atom energetics for predicting encapsulation of metal nanoparticles at the graphite surface. This is appealing, because calculations involving single metal atoms are generally simpler and less expensive than calculations involving metal clusters or metal slabs. It also supports a model in which the density of a two-dimensional gas of metal atoms beneath the graphite layer is key, leading to nucleation, growth, and trapping of metal nanoclusters.

We have also shown that the optimal temperature of metal deposition scales well with the cohesive energy of the metal, and can be used as a predictive tool. This is important in designing searches for metal encapsulation at graphitic surfaces. Presumably, this scaling reflects the necessity of metal cluster dissolution at defect sites, which maintains open portals during metal deposition.

\section{ACKNOWLEDGMENTS}

This work was supported by the U.S. Department of Energy (DOE), Office of Science, Basic Energy Sciences, Materials Sciences and Engineering Division. Research was performed at the Ames Laboratory, which is operated by Iowa State University under Contract No. DE-AC02-07CH11358. The experimental work was performed or supervised by A.L.-R., D.J., M.C.T., and P.A.T. DFT calculations were performed by Y.H., mainly using a grant of computer time at the National Energy Research Scientific Computing Centre (NERSC). NERSC is a DOE Office of Science User Facility supported by the Office of Science of the U.S. DOE under Contract No. DE-AC02-05CH11231. The calculations also partly used the Extreme Science and Engineering Discovery Environment (XSEDE), which is supported by the National Science Foundation under Grant No. ACI-1548562.

The authors declare no competing interests.
[1] S. E. Julien, A. Lii-Rosales, K. -T. Wan, Y. Han, M. C. Tringides, J. W. Evans, and P. A. Thiel, Squeezed nanocrystals: Equilibrium configuration of metal clusters embedded beneath the surface of a layered material, Nanoscale 11, 6445 (2019).

[2] A. Lii-Rosales, Y. Han, S. E. Julien, O. Pierre-Louis, D. Jing, K.-T. Wan, M. C. Tringides, J. W. Evans, and P. A. Thiel, Shapes of Fe nanocrystals encapsulated at the graphite surface, New J. Phys. 22, 023016 (2020).

[3] Q. Fu and X. H. Bao, Surface chemistry and catalysis confined under two-dimensional materials, Chem. Soc. Rev. 46, 1842 (2017).

[4] Y. Wang, J. Mao, X. G. Meng, L. Yu, D. H. Deng, and X. H. Bao, Catalysis with two-dimensional materials confining single atoms: Concept, design, and applications, Chem. Rev. 119, 1806 (2019).

[5] P. Gargiani, R. Cuadrado, H. B. Vasili, M. Pruneda, and M. Valvidares, Graphene-based synthetic antiferromagnets and ferrimagnets, Nat. Commun. 8, 699 (2017).

[6] V. G. Kravets, R. Jalil, Y. J. Kim, D. Ansell, D. E. Aznakayeva, B. Thackray, L. Britnell, B. D. Belle, F. Withers, I. P. Radko et al., Graphene-protected copper and silver plasmonics, Sci. Rep. 4, 5517 (2014).

[7] Y. Zhou, A. Lii-Rosales, M. Kim, M. Wallingford, D. Jing, M. C. Tringides, C. -Z. Wang, and P. A. Thiel, Defect-mediated, thermally-activated encapsulation of metals at the surface of graphite, Carbon 127, 305 (2018).

[8] A. Lii-Rosales, Y. Han, J. W. Evans, D. Jing, Y. Zhou, M. C. Tringides, M. Kim, C. -Z. Wang, and P. A. Thiel, Formation of multilayer $\mathrm{Cu}$ islands embedded beneath the surface of graphite: Characterization and fundamental insights, J. Phys. Chem. C 122, 4454 (2018).

[9] A. Lii-Rosales, Y. Han, K. M. Yu, D. Jing, N. Anderson, D. Vaknin, M. C. Tringides, J. W. Evans, M. S. Altman, and P. 
A. Thiel, Reverse-engineering of graphene on metal surfaces: A case study of embedded ruthenium, Nanotechnology 29, 505601 (2018).

[10] A. Lii-Rosales, Y. Han, K. C. Lai, D. Jing, M. C. Tringides, J. W. Evans, and P. A. Thiel, Fabricating Fe nanocrystals via encapsulation at the graphite surface, J. Vac. Sci. Technol. A 37, 061403 (2019).

[11] Y. Han, A. Lii-Rosales, Y. Zhou, C. J. Wang, M. Kim, M. C. Tringides, C.-Z. Wang, P. A. Thiel, and J. W. Evans, Nucleation and growth kinetics for intercalated islands during deposition on layered materials with isolated pointlike surface defects, Phys. Rev. Mater. 1, 053403 (2017).

[12] D. Appy, H. Lei, C.-Z. Wang, M. C. Tringides, D.-J. Liu, J. W. Evans, and P. A. Thiel, Transition metals on the (0001) surface of graphite: Fundamental aspects of adsorption, diffusion, and morphology, Prog. Surf. Sci. 89, 219 (2014).

[13] Y. Han, A. Lii-Rosales, M. C. Tringides, J. W. Evans, and P. A. Thiel, Energetics of copper adsorption and intercalation at graphite step edges, Phys. Rev. B 99, 115415 (2019).

[14] M. S. Dresselhaus and G. Dresselhaus, Intercalation compounds of graphite, Adv. Phys. 51, 1 (2002).

[15] G. A. Somorjai, Introduction to Surface Chemistry and Catalysis (John Wiley and Sons, New York, 1994).

[16] A. S. K. Hashmi and G. J. Hutchings, Gold catalysis, Angew. Chem. 45, 7896 (2006)

[17] P. Christopher and S. Linic, Engineering selectivity in heterogeneous catalysis: Ag nanowires as selective ethylene epoxidation catalysts, J. Am. Chem. Soc. 130, 11264 (2008).

[18] J. Zhao, A. O. Pinchuk, J. M. McMahon, S. Li, L. K. Ausman, A. L. Atkinson, and G. C. Schatz, Methods for describing the electromagnetic properties of silver and gold nanoparticles, Acc. Chem. Res. 41, 1710 (2008).

[19] L. Brus, Noble metal nanocrystals: Plasmon electron transfer photochemistry and single-molecule Raman spectroscopy, Acc. Chem. Res. 41, 1742 (2008).

[20] See Supplemental Material at http://link.aps.org/supplemental/ 10.1103/PhysRevResearch.2.033175 for more information on experimental details, calculation of relative error in island densities, and quantitative values of Pt cluster dimensions and densities.

[21] G. Kresse and J. Furthmüller, Efficient iterative schemes for ab initio total-energy calculations using a plane-wave basis set, Phys. Rev. B 54, 11169 (1996).

[22] G. Kresse and D. Joubert, From ultrasoft pseudopotentials to the projector augmented wave method, Phys. Rev. B 59, 1758 (1999).

[23] J. Klimes, D. R. Bowler, and A. Michaelides, Chemical accuracy for the van der Waals density functional, J. Phys.: Condens. Matter 22, 022201 (2010).

[24] D. D. L. Chung, Review graphite, J. Mater. Sci. 37, 1475 (2002).

[25] Y. Han, K. C. Lai, A. Lii-Rosales, M. C. Tringides, J. W. Evans, and P. A. Thiel, Surface energies, adhesion energies, and exfoliation energies relevant to copper-graphene and coppergraphite systems, Surf. Sci. 685, 48 (2019).

[26] D. Shoenberg, Fermi surfaces of Copper, Silver and Gold I. The de Haas-van Alphen effect, Philos. Trans. R. Soc., A 255, 85 (1962).

[27] C. Kittel, Introduction to Solid State Physics (John Wiley \& Sons, New York, 1996).
[28] J. W. Arblaster, Crystallographic properties of platinum, Platinum Metals Rev. 41, 12 (1997).

[29] M. Ziatdinov, S. Fujii, K. Kusakabe, M. Kiguchi, T. Mori, and T. Enoki, Direct imaging of monovacancy-hydrogen complexes in a single graphitic layer, Phys. Rev. B 89, 155405 (2014).

[30] J. R. Hahn and H. Kang, Vacancy and interstitial defects at graphite surfaces: Scanning tunneling microscopic study of the structure, electronic property, and yield for ion-induced defect creation, Phys. Rev. B 60, 6007 (1999).

[31] J. C. M. López, M. C. G. Passeggi, Jr., and J. Ferrón, Surface superstructures in highly oriented pyrolytic graphite surfaces after $\mathrm{Ar}_{+}$bombardment, Surf. Sci. 602, 671 (2008).

[32] S. Habenicht, K. P. Lieb, W. Bolse, U. Geyer, F. Roccaforte, and C. Ronning, Ion beam erosion of graphite surfaces studied by STM: Ripples, self-affine roughening and near-surface damage accumulation, Nucl. Instrum. Methods Phys. Res., Sect. B 161, 958 (2000).

[33] S. Habenicht, W. Bolse, H. Feldermann, U. Geyer, H. Hofsäß, K. P. Lieb, and F. Roccaforte, Ripple topography of ion-beameroded graphite: A key to ion-beam-induced damage tracks, EPL 50, 209 (2000).

[34] H. A. Mizes and J. S. Foster, Long-range electronic perturbations caused by defects using scanning tunneling microscopy, Science 244, 559 (1989).

[35] J. G. Kushmerick, K. F. Kelly, H. P. Rust, N. J. Halas, and P. S. Weiss, Observations of anisotropic electron scattering on graphite with a low-temperature scanning tunneling microscope, J. Phys. Chem. B 103, 1619 (1999).

[36] E. Sutter, D. P. Acharya, J. T. Sadowski, and P. Sutter, Scanning tunneling microscopy on epitaxial bilayer graphene on ruthenium (0001), Appl. Phys. Lett. 94, 133101 (2009).

[37] S. Marchini, S. Günther, and J. Wintterlin, Scanning tunneling microscopy of graphene on $\mathrm{Ru}(0001)$, Phys. Rev. B 76, 075429 (2007).

[38] E. Voloshina, N. Berdunov, and Y. Dedkov, Restoring a nearly free-standing character of graphene on $\mathrm{Ru}(0001)$ by oxygen intercalation, Sci. Rep. 6, 20285 (2016).

[39] S. J. Altenburg, J. Kroger, B. Wang, M.-L. Bocquet, N. Lorente, and R. Berndt, Graphene on Ru(0001): Contact Formation and Chemical Reactivity on the Atomic Scale, Phys. Rev. Lett. 105 , 236101 (2010).

[40] M. Gao, Y. Pan, L. Huang, H. Hu, L. Z. Zhang, H. M. Guo, S. X. Du, and H.-J. Gao, Epitaxial growth and structural property of graphene on $\mathrm{Pt}(111)$, Appl. Phys. Lett. 98, 033101 (2011).

[41] P. Merino, M. Švec, A. L. Pinardi, G. Otero, and J. A Martín-Gago, Strain-driven moiré superstructures of epitaxial graphene on transition metal surfaces, ACS Nano 5, 5627 (2011).

[42] C. M. Sung and M. F. Tai, Reactivities of transition metals with carbon: Implications to the mechanism of diamond synthesis under high pressure, Int. J. Refract. Met. Hard Mater. 15, 237 (1997).

[43] S. R. Shatynski, The thermochemistry of transition metal carbides, Oxid. Met. 13, 105 (1979).

[44] Y. Han, M. C. Tringides, J. W. Evans, and P. A. Thiel, Adsorption, intercalation, diffusion, and adhesion of $\mathrm{Cu}$ at the $2 \mathrm{H}-\mathrm{MoS}_{2}(0001)$ surface from first-principles calculations, Phys. Rev. Res. 2, 013182 (2020). 